\title{
Resistive MHD jet simulations with large resistivity
}

\author{
Miljenko Čemeljić, Jose Gracia, Nektarios Vlahakis and Kanaris Tsinganos
}

\begin{abstract}
Axisymmetric resistive MHD simulations for radially self-similar initial conditions are performed, using the NIRVANA code. The magnetic diffusivity could occur in outflows above an accretion disk, being transferred from the underlying disk into the disk corona by MHD turbulence (anomalous turbulent diffusivity), or as a result of ambipolar diffusion in partially ionized flows. We introduce, in addition to the classical magnetic Reynolds number Rm, which measures the importance of resistive effects in the induction equation, a new number $\mathrm{Rb}$, which measures the importance of the resistive effects in the energy equation. We find two distinct regimes of solutions in our simulations. One is the low-resistivity regime, in which results do not differ much from ideal-MHD solutions. In the high-resistivity regime, results seem to show some periodicity in time-evolution, and depart significantly from the ideal-MHD case. Whether this departure is caused by numerical or physical reasons is of considerable interest for numerical simulations and theory of astrophysical outflows and is currently investigated.
\end{abstract}




\section{Introduction}

In Vlahakis \& Tsinganos (1998) general classes of self-consistent ideal-MHD solutions have been constructed. In Vlahakis et al. (2000) Blandford \& Payne (1982) model was analysed, and the problem with the terminal wind solution (which was not causally disconnected from the disk) has been solved. The common deficiency of all radially self-similar models, a cut-off of the solution at small cylindrical radii and also at some finite height above the disk because of a strong Lorentz force close to the system's axis has been corrected numerically. A search in the numerical sim-
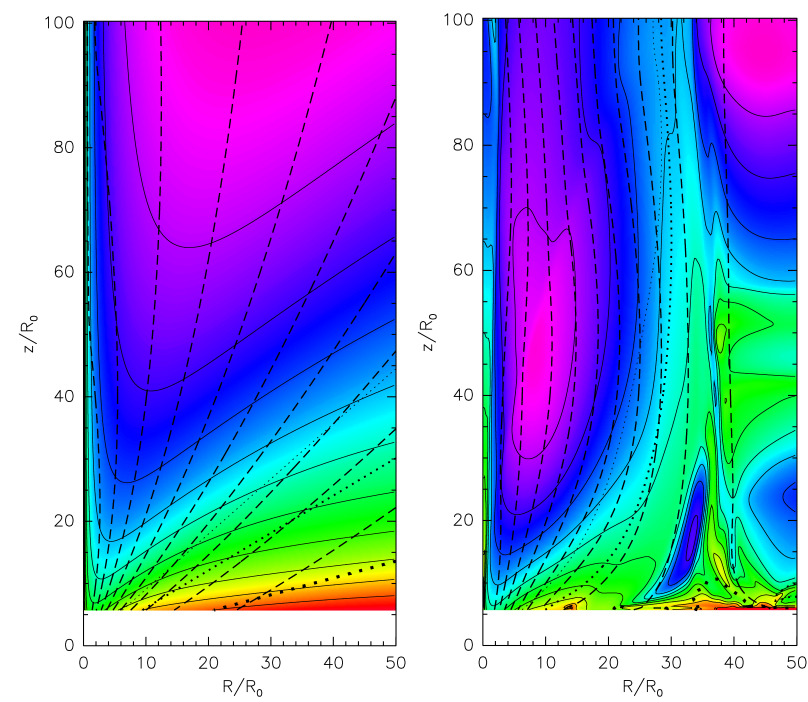

Fig. 1 The initial setup, which is slightly modified analytical solution, is shown in the Left panel. The solid lines represent logarithmically spaced isocontours of density. It is also shown in colour grading, in red to violet colour, for the logarithm of density -1 to -4 , respectively. In the Right panel shown is, in the same grading, the solution with large magnetic diffusivity. It does not reach stationary state, and shows some periodicity in time evolution. The dashed lines depict poloidal magnetic field lines, and the dotted lines depict the fast magnetosonic, Alfven and slow magnetosonic critical surfaces, top to bottom, respectively.

Miljenko Čemeljić

TIARA, Academia Sinica, National Tsing Hua University, No. 101, Sec. 2, Kuang Fu Rd., Hsinchu 30013, Taiwan e-mail:miki@tiara.sinica.edu.tw

Jose Gracia

School of Cosmic Physics, Dublin Institute for Advanced Studies, 31 Fitzwilliam Place, Dublin 4, Ireland e-mail:jgracia@cp.dias.ie

Nektarios Vlahakis \& Kanaris Tsinganos

IASA and Section of Astrophysics, Astronomy and Mechanics, Dpt. of Physics, Univ. of Athens, Panepistemiopolis 15784 Zografos, Athens, Greece e-mail: vlahakis, tsingan@phys.uoa.gr 
ulations for solutions at larger distances from the disk has been performed in Gracia et al. (2006) with NIRVANA code (version 2.0, Ziegler, 1998), and similar results were obtained also using the PLUTO code in Matsakos et al. (2008). Extension in the resistive-MHD has been investigated in Čemeljić et al. (2008) using the NIRVANA code and some of results we present here. Results of these investigations could also have implications in the numerical simulations of magnetospheric interaction in vicinity of the young stellar objects, where the resistivity plays important role.

Our numerical simulations are initiated by the slightly modified analytical solutions for radially self-similar flow from Vlahakis et al. (2000), and then evolved in the resistive MHD simulations by NIRVANA code. Our initial setup is shown in the left panel of Fig, 1

\section{New characteristic number}

In addition to the magnetic Reynolds number $\mathrm{Rm}=\mathrm{VR} / \eta$, which describes influence of the magnetic diffusivity $\eta$ in the induction equation, we introduced a new number, which describes the influence in the energy transport equation-see Fig 2 It can be written in terms of $\mathrm{Rm}$ and plasma beta as $\mathrm{Rb}=\mathrm{Rm} \beta / 2$. It is the ratio of the pressure term over the Joule heating term in the energy equation. When $\mathrm{Rb}$ is smaller or close to unity, which can happen even when Rm is much larger than unity, the energy dissipation becomes important. It might define one additional mode of resistive-

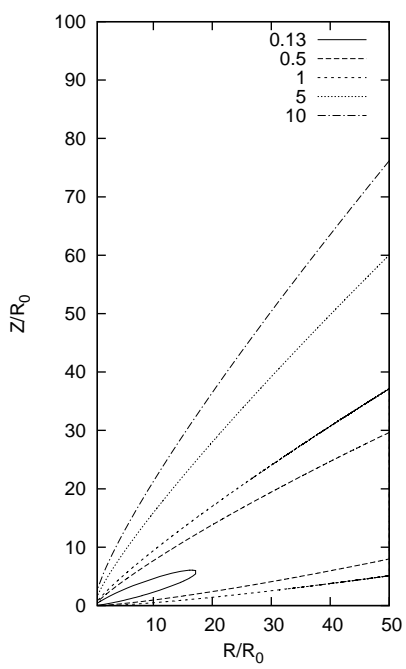

Fig. 2 Value of $\beta / 2\left(V R / V_{0} R_{0}\right)$ for the analytical solution used as initial setup here. This quantity gives the critical value of magnetic diffusivity $\eta$ that corresponds to $\mathrm{Rb}=1$. 
MHD solutions, indicated in our search for eventual onset of super-critical resistive regime.

\section{Results}

The resistive MHD jets are similar to ideal-MHD solutions for a finite range of magnetic diffusivity, in which they reach a well defined stationary state. This state only slightly differs from the initial state, as expected, since the initial setup was slightly modified analytical stationary solution. Departure from the ideal-MHD regime occurs for larger values of magnetic diffusivity, above some critical value. One such
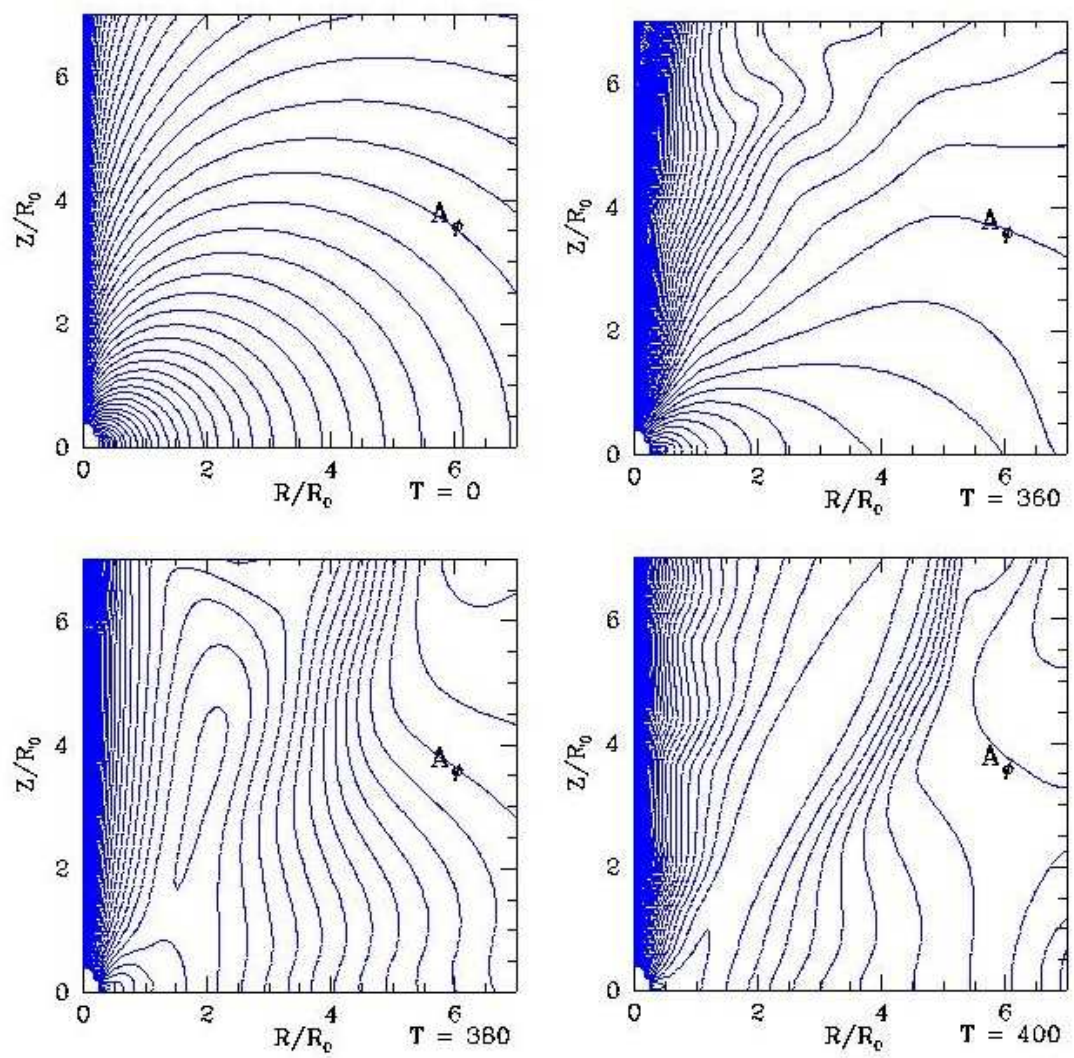

Fig. 3 Reconnection and re-shaping of the magnetic field in the vicinity of the young stellar object. Initially pure stellar dipole field reshapes into the stellar and disk open field during the timeevolution. The time is measured in the number of rotations at the inner disk radius, which is at $\mathrm{R}_{i}=3.0$ in these simulations. Without the substantial resistivity, reconnection does not occur and simulations terminate because of numerical reasons. 
result is shown in the right panel of Fig 1 We note possible existence of the distinct super-critical regime in magnetic diffusivity in our simulation for the outflows initialised with a self-similar analytical solutions. We also define the new characteristic number, which describes the influence of the resistivity on the energy transport equation. Physical parameters and the eventual periodicity of the super-critical resistive solutions are currently under investigation. Such solutions might be interesting for investigations of accretion flows in the vicinity of young stellar objects, where the magnetic resistivity seems to play important role.

In the Fig 3 we show one case in numerical simulations of magnetospheric interaction in the closest vicinity of young stellar object (Čemeljić et al., 2009). These simulations have been performed with code ZEUS347, which is our resistive version of Zeus-3D code (Fendt \& Čemeljić, 2002). Magnetic reconnection shows to play essential role in re-shaping the initial stellar dipole, which enables the launching of outflows. In our numerical simulations, if the resistivity is too small, reconnection will not occur. Therefore, we need to investigate parameter space for resistivity, and we need to understand what are the effects of large, and not only negligible or very small resistivity.

Acknowledgements This work was supported in part by EC's Marie Curie Actions-Human Resource and Mobility within the JETSET network under contract MRTN-CT-2004005592. MČ expresses gratitude to TIARA/ASIAA in Taiwan for possibility to use their Linux clusters and JETSET for supporting this collaboration.

\section{References}

1. Blandford R. D., Payne D. G., 1982, MNRAS, 199, 883

2. Čemeljić M., Hsien S., Chiang T.-Y., 2009, in preparation

3. Čemeljić M., Gracia J., Vlahakis N., Tsinanos K., 2008, MNRAS, 389, 1022, 1032

4. Fendt Ch., Čemeljić M., 2002, A\&A, 395, 1045

5. Gracia J., Vlahakis N., Tsinganos K., 2006, MNRAS, 367, 201

6. Matsakos T., Tsinganos K., Vlahakis N., Massaglia S., Trussoni E., 2008, A\&A, 477, 521

7. Vlahakis N., Tsinganos K., 1998, MNRAS, 298, 777

8. Vlahakis N., Tsinganos K., Sauty C., Trussoni E., 2000, MNRAS, 318, 417

9. Ziegler U., 1998, Comput. Phys. Commun., 109, 111 